\title{
Knockdown CD44 promote the inflammatory response through the autophagy pathway in mouse models of pulmonary contusion
}

\author{
Songlin Yang ${ }^{1}$, Xianglin Meng ${ }^{1}$, Wei Yu ${ }^{1}$, Dongsheng Fei ${ }^{1}$, Wei Yang ${ }^{1}$, Shishuai Meng ${ }^{1}$, Peiyao Luo ${ }^{1}$, \\ Jianpeng Wang ${ }^{1}$, Shangha Pan ${ }^{2}$, Mingyan Zhao' \\ ${ }^{1}$ Department of ICU, the First Affiliated Hospital of Harbin Medical University, Harbin, China; ${ }^{2}$ The Key Hepatosplenic Surgery Laboratory, \\ Department of General Surgery, the First Affiliated Hospital of Harbin Medical University, Harbin, China \\ Contributions: (I) Conception and design: S Yang, M Zhao; (II) Administrative support: S Yang, X Meng, W Yu; (III) Provision of study materials \\ or patients: D Fei, W Yang, S Meng, P Luo, J Wang, S Pan, S Yang; (IV) Collection and assembly of data: S Yang, M Zhao; (V) Data analysis and \\ interpretation: D Fei, W Yang, S Meng, P Luo, J Wang, S Pan, S Yang; (VI) Manuscript writing: All authors; (VII) Final approval of manuscript: All \\ authors. \\ Correspondence to: Mingyan Zhao. Department of ICU, the First Affiliated Hospital of Harbin Medical University, Harbin, China. \\ Email: mingyan197@gmail.com.
}

Background The effects of CD44, via the anti-inflammatory functions of autophagy, on lung injuries following pulmonary contusion (PC) and cell apoptosis were investigated.

Methods: Acute lung injury (ALI) mouse models were established by inducing lung injury via PC. This injury was verified using hematoxylin and eosin (H\&E) staining, following which bronchoalveolar lavage fluid (BALF) was collected from these mice for analysis and further experimentation. CD44, LC3 I/II ratio, Beclin-1, and p62 expression levels in A549 cells were determined using immunohistochemistry, and western blot assays. CCK-8, flow cytometry, and acridine orange/ethidium bromide (AO/EB) fluorescence staining were used to quantify cell growth induced by BALF. LC3 II and LC3 I expression was determined through immunofluorescence. CD44-knockdown mice were used to demonstrate lung function after PC.

Results: The successful establishment of the ALI mouse models, created via PC was confirmed by an enhanced inflammatory response in the lung tissue, markers of cell autophagy. The ALI mice were found to have elevated CD44 expression. The viability of A549 cells exposed to BALF was downregulated, while the knockdown of CD44 promoted this effect. AO/EB and flow cytometry also indicated that the knockdown of CD44 promoted the cell apoptosis induced by BALF. Western blot analysis showed that knockdown of CD44 can inhibit LC3 I/II, p62, and Beclin-1 expression induced by BALF exposure. Additionally, knockdown of CD44 in mice was found to promote PC-induced lung injury through the attenuation of autophagy.

Conclusions: Knockdown CD44 was shown to inhibit cell growth and induced cell apoptosis via autophagy signaling pathways, promote mice with ALI induced by PC in vivo and in vitro.

Keywords! CD44; pulmonary contusion (PC); autophagy; apoptosis

Submitted Feb 11, 2020. Accepted for publication Sep 18, 2020.

doi: 10.21037/apm-20-378

View this article at: http://dx.doi.org/10.21037/apm-20-378

\section{Introduction}

Every year approximately 5.8 million patients are hospitalized and die from multiple trauma, making multiple trauma the leading cause of death in the world (1-3).
Intensive care unit (ICU) treatment is required by $37.5 \%$ of patients with multiple trauma (4). Of patients with multiple trauma, those with chest trauma have the thirdhighest mortality rate after patients with brain trauma and abdominal trauma. Chest trauma often leads to pulmonary 
contusion (PC). Patients with PC account for approximately $30 \%$ of hospitalized multiple trauma patients, and the PC patient mortality rate is as high as $10-25 \%(5,6)$. As an independent risk factor for acute respiratory distress syndrome (ARDS) and ventilator-associated pneumonia, PC prolongs the duration of hospital stay, increases patient medical costs, and is also closely related to patient mortality $(7,8)$. Cytokines, the complement system, and the coagulation system are involved in activating inflammation following trauma. This persistent excessive inflammatory response after trauma is closely related to the occurrence of acute lung injury (ALI) (9).

CD44 is a cell surface glycoprotein involved in cell migration and adhesion, as well as cell-cell interactions. This glycoprotein has been found to be directly involved in the development of multiple diseases, including lung inflammation (10-12). The binding of CD44-specific monoclonal antibodies (mAbs) to CD44, for instance, can trigger lymphocyte proliferation $(13,14)$. This is comparable to interleukin (IL)-2-dependent lymphocyte proliferation observed in cells stimulated with anti-CD3 mAbs. CD44 binding has also been found to be involved in natural killer (NK) 2 activation by increasing NK-mediated cell lysis of NK-sensitive targets (15). The importance of CD44 in the inflammatory response is supported by numerous studies $(13,16,17)$.

Autophagy is a highly conserved intracellular degradation process and is a cell protection mechanism (18). Multiple stress factors, such as damage caused by trauma-related inflammation, hyperoxia, and sepsis, may induce autophagy and contribute to the pathogenesis of ALI. An example that supports the involvement of autophagy in ALI is that 3-methyladenine, an autophagy inhibitor, provides protection against endothelial cell barrier dysfunction in this condition (19). Additionally, autophagy has been found to be involved in rapamycin-mediated LPS-induced ALI in rats (20). Autophagy has also been shown to be an essential component of ALI, but its role and mechanism in PCinduced lung injury have yet to be conclusively determined.

The hypothesis tested in this study was that CD44 activation results in increased autophagy, indicating that CD44 plays a vital role in the development of PC-induced lung injury. We further examined the role and mechanism of CD44 knockdown in A549 cells exposed to the bronchoalveolar lavage fluid (BALF) of ALI mouse models, as well as how CD44 knockdown mice respond to lung injury. This study provided insights that further develop the understanding of the role of CD44 in PC-induced lung injury. We present the following article in accordance with the ARRIVE reporting checklist (available at http://dx.doi. org/10.21037/apm-20-378).

\section{Methods}

\section{Differential expression analysis of genes in multiple trauma}

We downloaded the expression profile dataset GSE36809 from the Gene Expression Omnibus (GEO) database to analyze multiple trauma data. This contained the data of 820 severe blunt trauma samples and 37 control samples. After downloading the original data, we standardized and analyzed our data using R software affy and limma packages. Using a $|\log \mathrm{FC}|$ value of $>1$ (bottom 2 ) and a $\mathrm{P}$ value of $<0.05$ for filtering, we obtained the differentially expressed genes (DEGs). The limma package was used to analyze the expression profile data to obtain the normalized expression level of each probe. The ggplot2 package was used to construct volcano plots.

\section{Animals}

Male C57BL/6 mice (Beijing Vital River Laboratory Animal Technology Co., Ltd., Beijing, China) and male CD44deficient (CD44-/-) mice (CD44 knockdown mice) (Model Animal Research Center of Nanjing University, Nanjing, China) that were aged matched from 8-12 weeks were used in this study. The Ethics Committee of Harbin Medical University (No. IRB-AF/SC-08/050, Heilongiiang, China) approved all experimental procedures. The procedures complied with all related state regulations.

\section{PC model}

The animals were first randomly assigned to the following experimental groups: the sham group, the PC group, the CD44 knockout group (the CD44-/- group), and the PC plus CD44 knockout group (the PC + CD44-/- group).

A cortical contusion impactor (CCI) was used to induce $\mathrm{PC}$ as described in previous literature (21). The mice were first anesthetized by an intraperitoneal injection of $60 \mathrm{mg} / \mathrm{kg}$ of pentobarbital sodium. Each mouse was placed in the prone position and the CCI was inserted along the posterior axillary line of the right chest, $1 \mathrm{~cm}$ above the costal margin. A precise quantity of energy $\left(152 \mathrm{~J} / \mathrm{m}^{2}\right)$ was applied to induce a reproducible PC. The sham mice only received 
anesthetic. The mice were sacrificed 24 hours after injury by means of pentobarbital overdose by intraperitoneal injection and cervical dislocation. BALF and lung tissue were collected at the time of death.

\section{Bronchoalveolar lavage and cytology}

After the mice were sacrificed, median sternotomy was used to expose the lungs and mouth of each mouse. An intravenous infusion needle was inserted into the exposed trachea. BALF was obtained via cannulation of the trachea with a 24-gauge feeding needle and serial lavage of the lungs using $1 \mathrm{~mL}$ of phosphate-buffered saline (PBS); this was done three times and the BALF from each procedure was combined.

\section{Histological analysis}

Lung tissues from the sacrificed mice were isolated and fixed overnight with $10 \%$ buffered formalin, following which they were embedded in paraffin. The samples were cut into 4- $\mu \mathrm{m}$ sections and stained using hematoxylin and eosin $(\mathrm{H} \& \mathrm{E})$. Histological evaluation of the tissue was performed using light microscopy, including evaluation of cellular infiltration. A semiquantitative scoring system described in previous literature was used to determine pathological severity response. This scoring system required information on alveolar wall thickness, aggregation of neutrophils or leukocyte infiltration, and hemorrhage and alveolar congestion, producing a grade of 0 (absent) to 4 (extensive) (22).

\section{Western blot analysis}

Western blot analysis was used to evaluate the expression of LC3, p62 and Beclin-1 in A549 cells after these cells had been exposed to BALF for 24 hours (15). To homogenize the cells, $20 \mathrm{mg}$ was mixed with $200 \mu \mathrm{L}$ of RIPA buffer (Shanghai Beyotime Biotechnology Co., Ltd., Shanghai, China), and then the membranes were blocked overnight with $5 \%$ skim milk at $4{ }^{\circ} \mathrm{C}$. This was followed by incubation with rabbit anti-human primary mAbs $(1: 1,000)$ at room temperature for 2 hours. After the membranes had been washed, the mixture was incubated for an hour with cationized horseradish peroxidase (CHRP)conjugated secondary antibodies (goat anti-rabbit 1:2,000 Zhongshanjinqiao, China). The membranes were then developed under ultraviolet light and examined with an image analyzing system.

\section{Acridine orange/ethidium bromide (AO/EB) fluorescence staining}

A549 cells were exposed to BALF and CD44-siRNA for 48 hours. The cells were then incubated for 5 minutes with AO/EB (Solarbio Biotechnology, China) at a scale of 1:1. The following formula was used to calculate the percentage of apoptotic cells: apoptotic rate $(\%)=$ number of apoptotic cells/total number of cells counted.

\section{Autophagic flux assessment using tandem mRFP-GFP-LC3}

Adenovirus carrying mRFP-GFP-LC3 was purchased from Hanbio Co., Ltd. (China). To examine autophagy flux, A549 cells were exposed to BALF and CD44-siRNA and then infected with mRFP-GFP-LC3 adenovirus. The medium was changed after 12 hours and fluorescence images were captured 24 hours after infection. Colocalization of GFP and $\mathrm{mRFP}$ fluorescence indicated that a compartment had not fused with a lysosome. An mRFP signal without GFP, however, indicated that a compartment had fused with a lysosome.

\section{Flow cytometry}

Apoptosis of the A549 cells treat with BALF; BALF + CD44-siRNA was determined using flow cytometry. Briefly, the transfected cells were reseeded into $6-\mathrm{cm}$ dishes at a density of $2 \times 10^{5}$ cells per dish. After 48 hours, the cells were digested using $0.25 \%$ trypsin. The cells were then centrifuged, collected, and fixed overnight using precooled $70 \%$ ethanol. To analyze apoptosis, the immobilized cells were incubated with $5 \mathrm{ll}$ Annexin V-FITC and $5 \mathrm{ll} \mathrm{PI}$ (Beyotime, China) for 20 minutes in the dark at $25^{\circ} \mathrm{C}$. A BD FACSCalibur Flow Cytometer (BD Biosciences, USA) was used for the analysis.

\section{Statistical analysis}

The statistical analyses were conducted using GraphPad Prism 8.0 software. The data are presented as mean \pm SD. Comparisons were conducted using analysis of variance and Student's $t$-tests. A P value of $<0.05$ was considered statistically significant. 


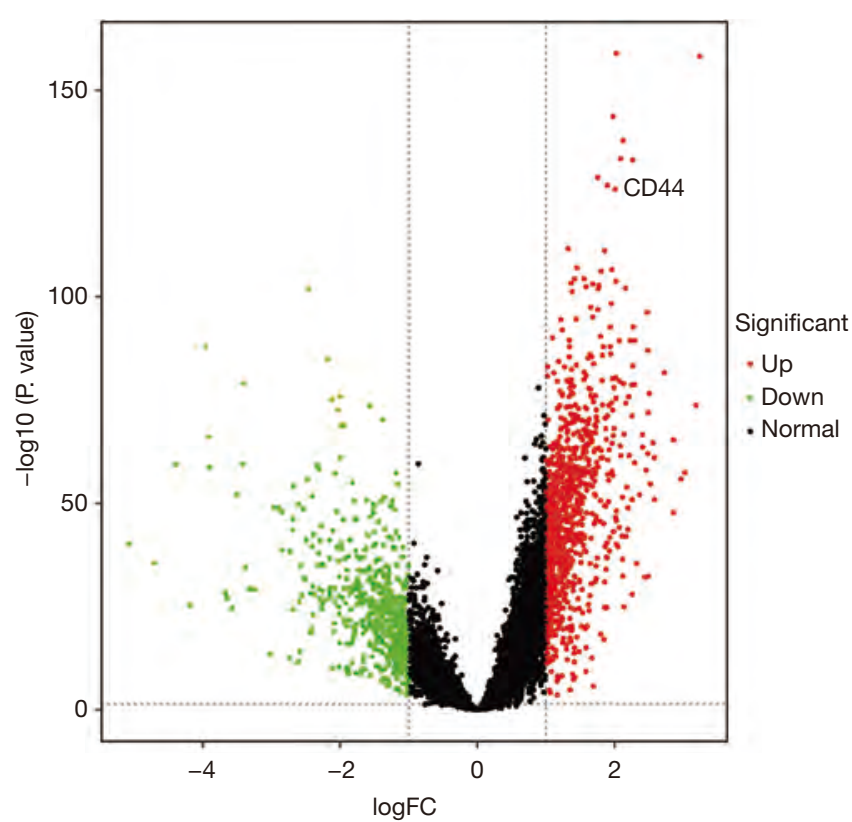

Figure 1 The expression of CD44 in multiple trauma patients and control patients expressed by bioinformatics.

\section{Results}

\section{CD44 gene expression in multiple trauma patients}

Of the DEGs found in trauma patients, we selected two subtypes of $C D 44$ genes for further analysis. We found that $C D 44$ gene expression in multiple trauma patients was significantly elevated compared with the control group (Figure 1) $(\mathrm{P}<0.01)$.

\section{Effects of CD44 on lung injury in mice with PC}

The H\&E staining of the sectioned lung tissue and following histological assessment revealed that the lung tissue was normal in both the sham group and the CD44-/group: the alveolar structure was complete and there was no interstitial edema, no inflammatory cell infiltration, no alveolar congestion, and no bleeding (Figure 2A). No significant difference in histological score was found between these two groups. In the PC group and the PC + CD44-/- group, the lung tissue had lost its normal morphology and the alveolar septum was significantly thickened. Additionally, there was increased infiltration of inflammatory cells and increased exudation of the alveolar cavity (Figure 2A). Compared with the sham group, the lung pathological scores of the PC group and PC + CD44-/- group increased significantly, and the PC + CD44-/- group showed more pronounced damage (Figure 2B). There was no significant difference between the protein concentration in the PC of the sham group and that of the CD44-/group. There was a significant difference, however, between the protein concentration in the $\mathrm{PC}$ of the sham group and that of the PC group, and an even greater significant difference between that of the sham group and that of the PC + CD44-/- group (Figure 2C).

\section{Effect of CD44 knockdown on BALF-induced injury in A549 cells}

To analyze the role of CD44 in A549 cells in vitro, we first determined the viability of A549 cells after BALF exposure in a time and concentration-dependent manner. The viability of A549 cells exposed to BALF decreased, verifying that BALF had caused injury to the cells (Figure S1). We then analyzed the cell growth of A549 cells after exposure to BALF, exposure to CD44-siRNA, or exposure to BALF and CD44-siRNA in combination. CD44-siRNA causes CD44 knockdown and, compared with the BALF group, cells exposed to BALF + CD44-siRNA demonstrated a decrease in cell growth (Figure $3 A$ ) and a decrease in the rate of apoptosis $(\mathrm{P}<0.01)$ (Figure 3B). Notably, flow cytometry performed on the A549 cells exposed to both BALF and BALF + CD44-siRNA indicated that CD44 knockdown increased BALF-induced cell apoptosis (Figure 3C). These results indicate that CD44-knockdown exerts apoptotic functions in BALF-induced injury to A549 cells.

\section{Effect of CD44 on expression of autophagy pathway proteins in cells exposed to BALF}

Autophagy analysis further showed that BALF can upregulate the expression of LC3 (Figure $4 A$ ). We then measured the expression levels of p62, Beclin-1, and LC3 after exposing A549 cells to either BALF or BALF and CD44-siRNA in combination. Cells exposed to BALF and CD44-siRNA in combination showed decreased expression levels of p62, compared with cells exposed only to BALF $(\mathrm{P}<0.01)$ (Figure $4 B)$. In cells exposed to CD44siRNA but not exposed to BALF, the expression levels of Beclin-1 and LC3 II/I were also reduced compared with cells exposed to BALF $(\mathrm{P}>0.05)$ (Figure $4 B$ ). Double staining immunohistochemistry for LC3 II/I also confirmed the results of protein result of LC3 II/I (Figure 4C). This 
A

Sham
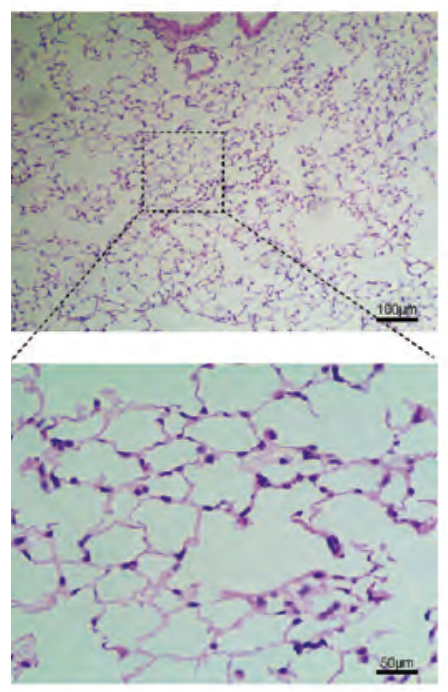

CD44-/-

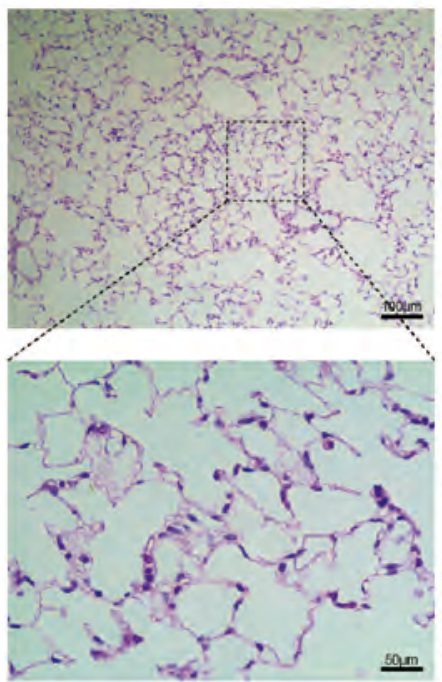

PC

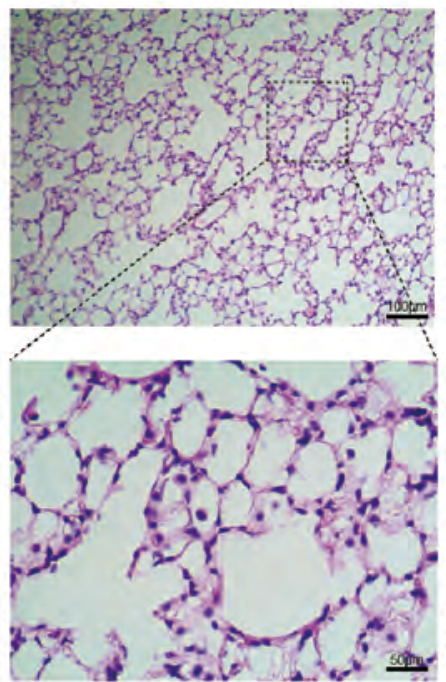

$\mathrm{PC}+\mathrm{CD} 44-/-$

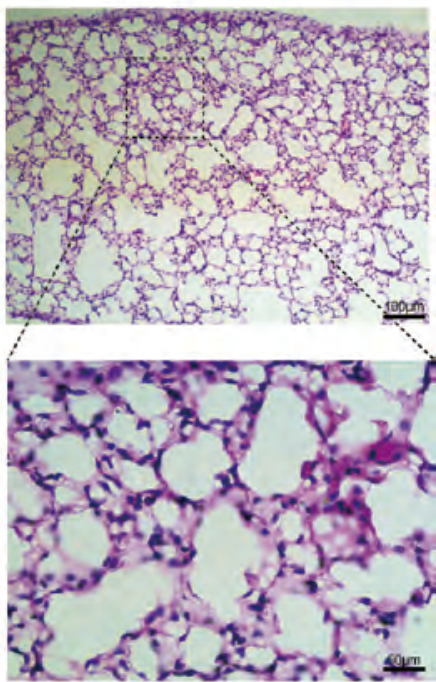

B

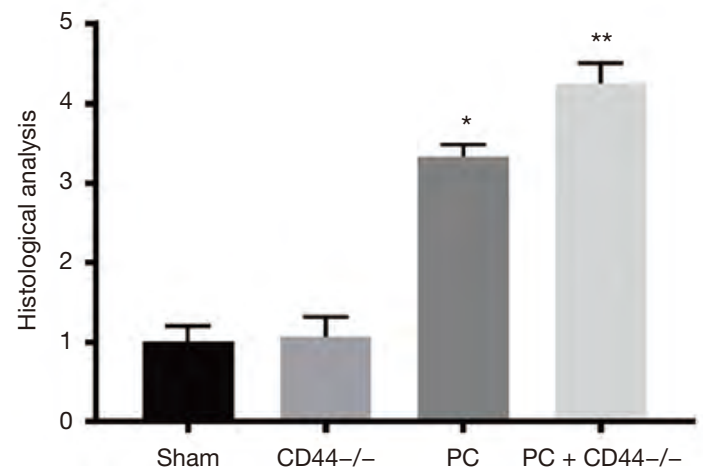

C

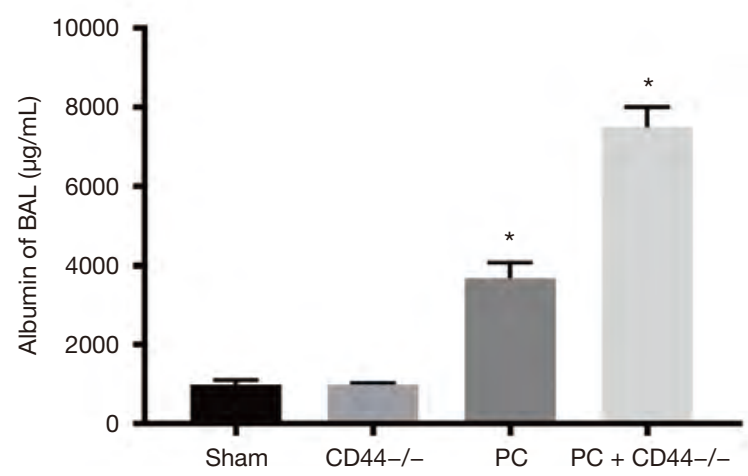

Figure 2 Effect of CD44 on lung injury in mice with PC. (A) H\&E staining of lung tissue from sham group, CD44-/- group, PC group, and PC + CD44-/- group mice; (B) lung pathological scores of the different groups of mice; (C) protein concentration in the BALF of mice. Results are presented as mean $\pm \mathrm{SD}, \mathrm{n}=8$ in each group. * $\mathrm{P}<0.05$ vs. the $\mathrm{PC}$ group; ${ }^{* *}, \mathrm{P}<0.01$ vs. the sham group. $\mathrm{CD} 44-/-, \mathrm{CD} 44$ knockdown; PC, pulmonary contusion; H\&E, hematoxylin and eosin; BALF, bronchoalveolar lavage fluid.

data suggests that CD44-knockdown promote BALFinduced injury in A549 cells by regulating the expression of autophagy pathway proteins.

\section{Effect of CD44 on PC mice lung tissue}

Autophagy relies heavily on Beclin-1, LC3, and p62. Immunohistochemical staining revealed the presence of these proteins in alveolar epithelial cells, vascular endothelial cells, and bronchial epithelial cells. LC3 was predominantly expressed in cell cytoplasm, particularly that of alveolar septal epithelial cells. Compared with the sham group, the PC group showed significantly increased levels of LC3 expression in the lung tissue. The expressions of Beclin-1, LC3, and p62 in the PC + CD44-/- group mice lung tissue were decreased compared with those in the tissue of the BALF group mice (Figure $5 A, B, C, D)$.

\section{Survival rate of mice}

To observe the effect of CD44 on the 7-day survival rate of mice with PCs, mice were divided into four groups: the sham 
A

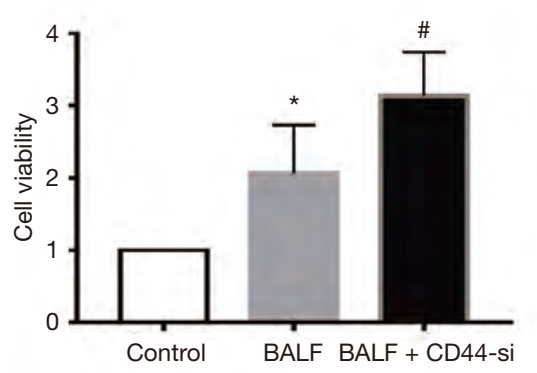

C
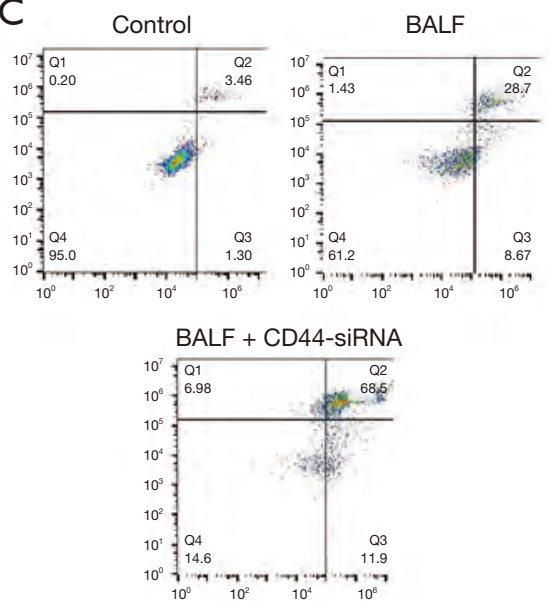

B

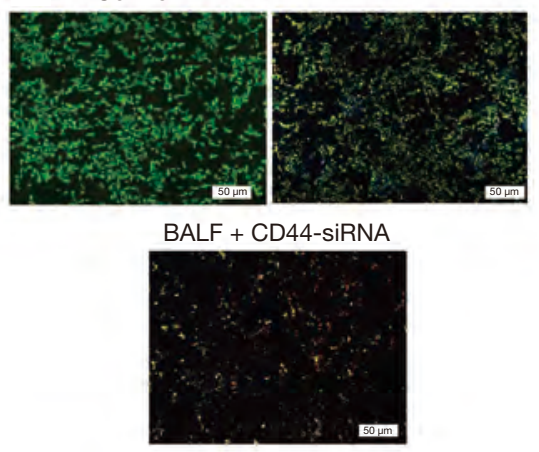

BALF

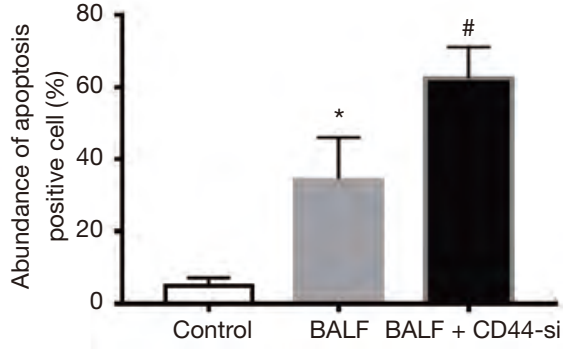

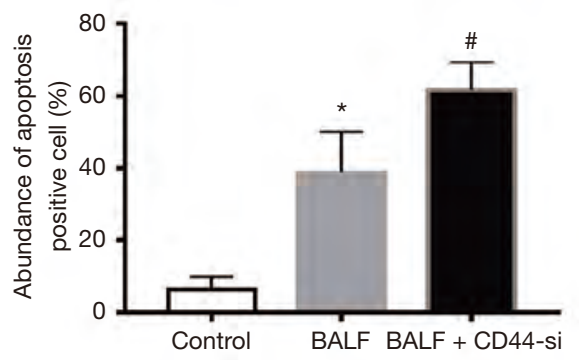

Figure 3 Knockdown of CD44 provides promotion from injury induced by BALF. (A) Cell viability; (B) AO/EB staining; (C) flow cytometry. Results are presented as mean $\pm \mathrm{SD}, \mathrm{n}=3-6$ in each group. *, $\mathrm{P}<0.05$ vs. the BALF group; ${ }^{*}, \mathrm{P}<0.01$ vs. the control group. BALF, bronchoalveolar lavage fluid; $\mathrm{AO} / \mathrm{EB}$, acridine orange/ethidium bromide.

group, the CD44-/- group, the PC group, and the PC + CD44-/- group. Each group comprised 10 mice (Figure 6). We observed no differences in survival rates between the CD44-/- group and the sham group. The survival rates of the PC group and the PC + CD44-/- groups, however, were significantly decreased $(\mathrm{P}<0.01)$, compared with the sham group. The mice in the $\mathrm{PC}+\mathrm{CD} 44-/-$ group showed higher mortality $(\mathrm{P}<0.05)$.

\section{Discussion}

Of patients that present with multiple trauma, more than $75 \%$ have chest trauma (23), and $25 \%$ of these patients require hospitalization. There is more than a 2.5 -fold increase in patient mortality, as well as duration of hospital stay, when multiple traumata is complicated by PC. ARDS caused by PC is different to that caused by other conditions, as oxygen treatment alone is not sufficient to relieve hypoxia; severely affected patients may require mechanical ventilation $(8,24)$.

The pathogenesis of PC is complicated (25). In addition to the direct injury, damaged tissue and detached extracellular matrix (ECM) can activate the innate immune system, causing local inflammation of lung tissue $(26,27)$. Subsequent activation and recruitment of neutrophils to the injured alveolar epithelial cells may lead to ARDS (28). The innate immune response directly contributes to the development of ARDS caused by PC (29). Changes in the ECM following lung injury can activate and recruit neutrophils by the release of cytokines and chemokines, which then promote interactions between immune and nonimmune lung cells. The trauma of the initial PC-causing injury can release damage-associated molecular patterns (DAMPs), such as hyaluronic acid, heparin sulfate, heat shock protein, HMGB-1, and mitochondrial DNA, which activate endothelial cells and inflammatory cells via TLR2, 
A

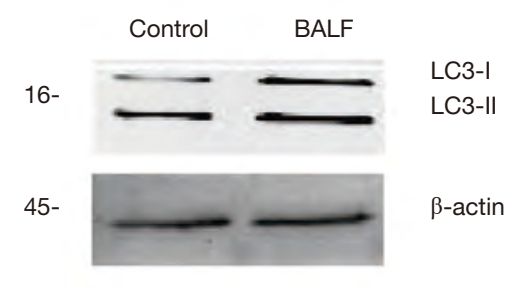

B

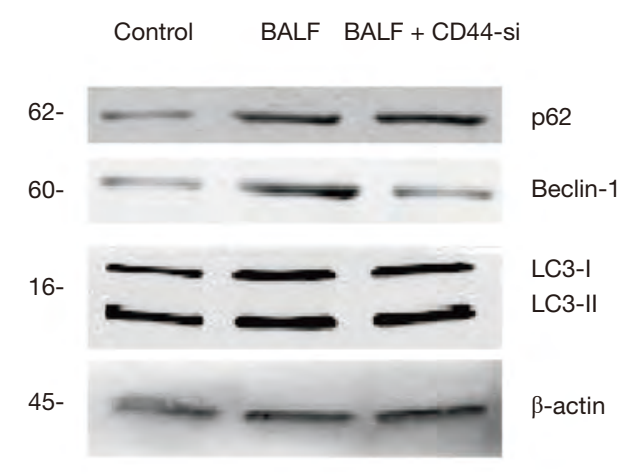

C Control

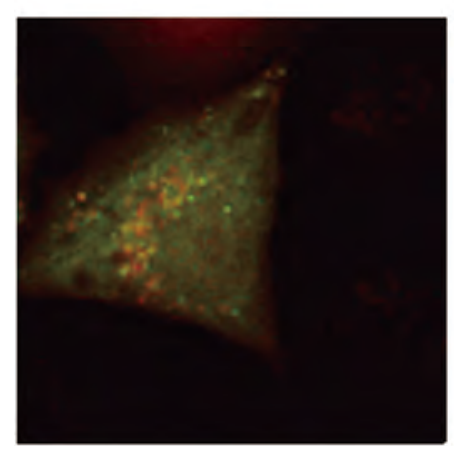

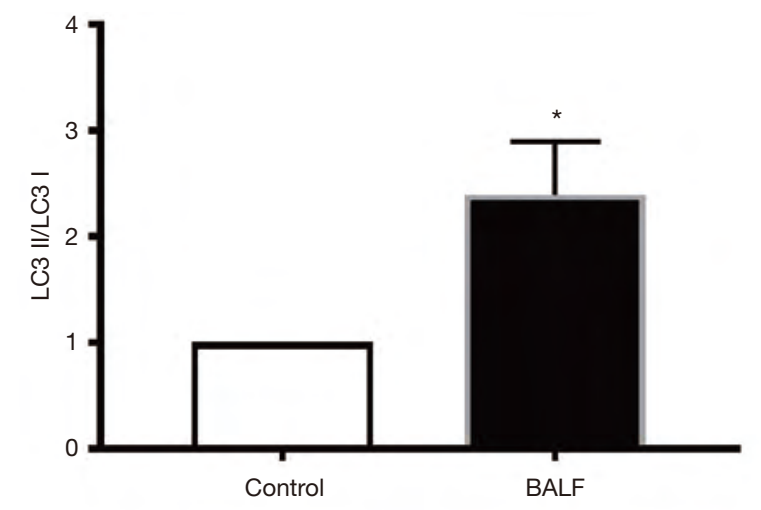

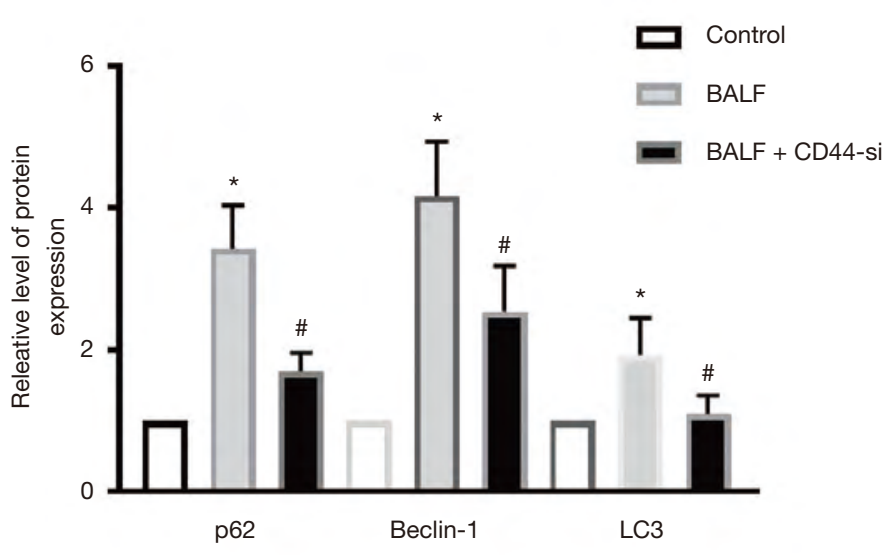

Figure 4 Effect of CD44 on BALF cell changes during autophagy. (A) LC3 levels after A549 cells were exposed to BALF; (B) levels of p62, Beclin-1, and LC3 after A549 cells were exposed to either BALF or BALF + CD44-siRNA; (C) immunofluorescence staining of LC3 after A549 cells were exposed to either BALF or BALF + CD44-siRNA. Scale bar, $50 \mu \mathrm{m}$. Data are presented as mean $\pm \mathrm{SD}, \mathrm{n}=3-6$ in each group. *, $\mathrm{P}<0.05$ vs. the control group; , $\mathrm{P}<0.01$ vs. the BALF group. BALF, bronchoalveolar lavage fluid.

TLR4, and CD44 located on cellular surfaces. This results in the release of cytokines and chemokines, recruitment of neutrophils, and activation of macrophages, which together lead to tissue damage.
CD44 is a type I transmembrane glycoprotein that is widely expressed on the surface of various lung cells. This glycoprotein can regulate immune function by mediating cell-to-cell and cell-to-ECM interactions $(30,31)$. We 

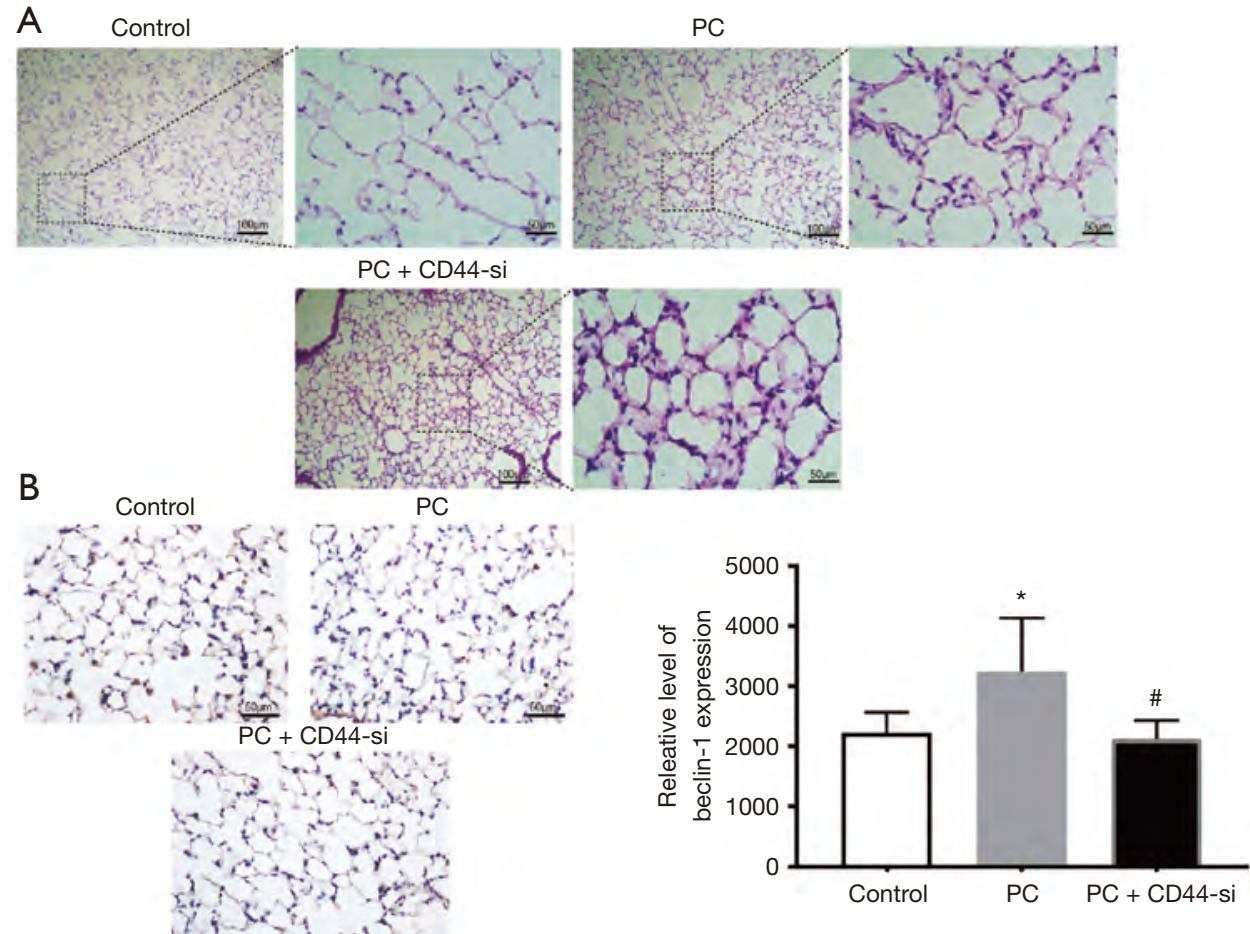

C
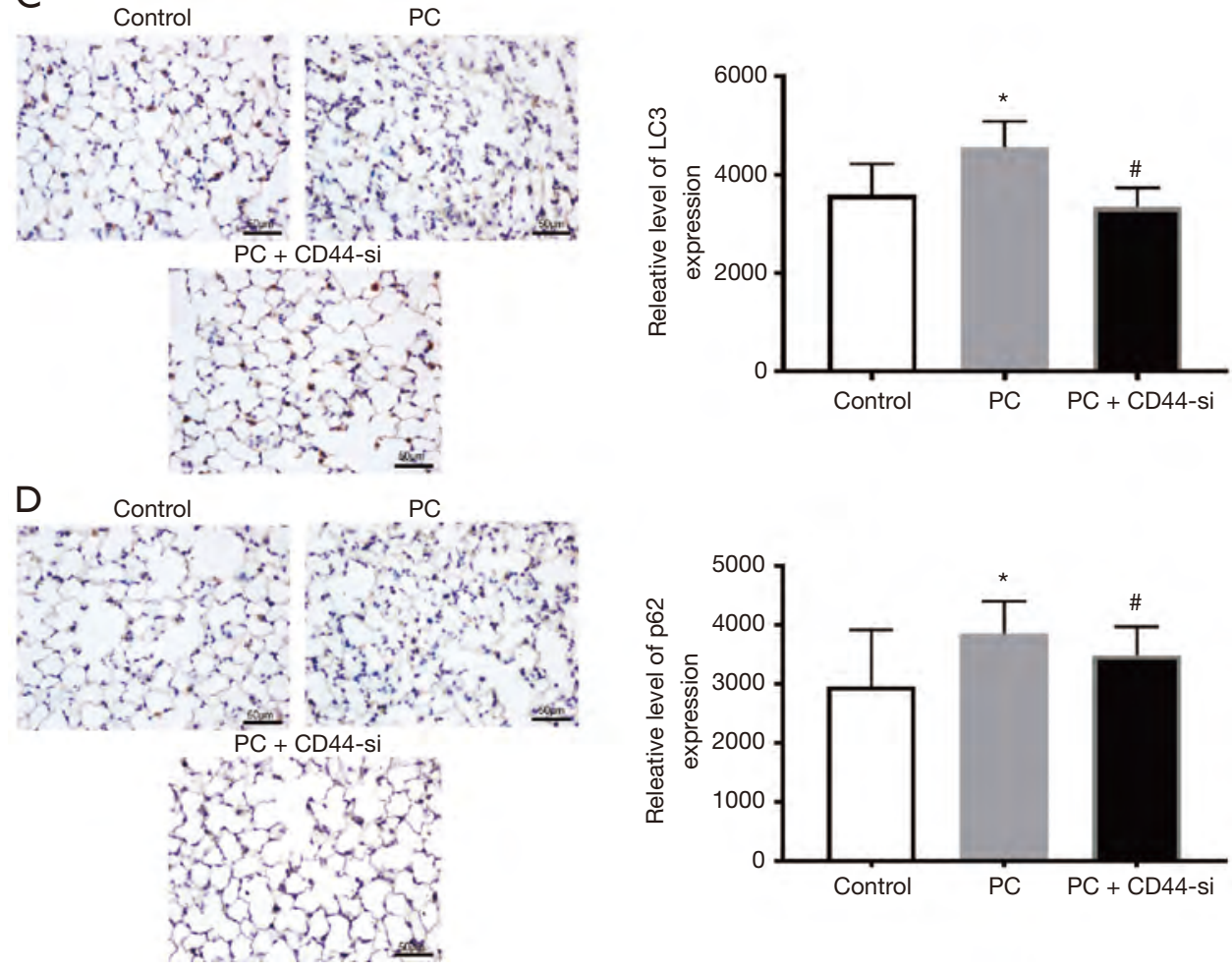

Figure 5 Effect of CD44 on autophagy in lung tissue of mice with PC. (A) H\&E staining of lung tissue from sham group, CD44-/- group, PC group, and PC + CD44-/- group mice; (B) Beclin-1 expression determined by immunohistochemistry; (C) LC3 expression determined by immunohistochemistry; (D) p62 expression determined by immunohistochemistry. Data are presented as mean $\pm \mathrm{SD}, \mathrm{n}=8$ in each group. *, $\mathrm{P}<0.05$ vs. the control group; ${ }^{\prime}, \mathrm{P}<0.01$ vs. the BALF group. CD44-/-, CD44 knockdown; PC, pulmonary contusion. 


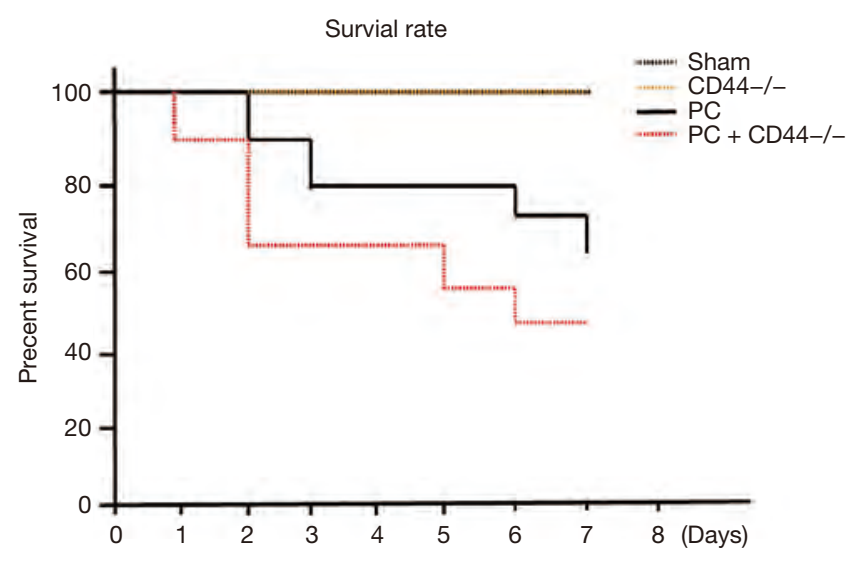

Figure 6 Seven-day survival curves of experimental mice groups. CD44-/-, CD44 knockdown; PC, pulmonary contusion.

analyzed data from trauma-related studies in the GEO database, which revealed significant differences in CD44 gene expression in trauma patients. Along with alveolar tissue destruction, the infiltration of immune cells to the lungs is another characteristic outcome of PC. In this study, using PC modeling we found that, 24 hours following contusion, mice belonging to the $\mathrm{PC}$ group and $\mathrm{PC}+$ CD44-/- group no longer had normal lung morphology compared with sham group mice. Additionally, the alveolar interval was significantly thickened, inflammatory cell infiltration had occurred, and alveolar exudation was evident in these groups compared with the sham group. The lung pathological scores of the PC group and PC + CD44-/group rose significantly, particularly for the $\mathrm{PC}+\mathrm{CD} 44-/-$ group. Compared with $\mathrm{PC}$ group mice, the protein concentration in $\mathrm{PC}$ of the $\mathrm{PC}+\mathrm{CD} 44-/-$ group increased more significantly.

Apoptosis is a common manifestation of damage that occurs during PC (32). This particularly occurs in type II alveolar epithelial cells, which further aggravates the initial damage caused to the lung and can lead to ARDS; the apoptosis of these cells is closely related to both the progression and repair of lung injury (33). Following type II alveolar epithelial cell apoptosis, pulmonary surfactant secretion decreases, causing increased alveolar surface tension and atelectasis (34). In addition to the direct damage caused by PC, neutrophils activated by this process can directly induce apoptosis in type II alveolar epithelial cells (35). In this study, we found that the lungs of mice in the PC + CD44-/- group showed significantly increased apoptosis. In experiments where A549 cells were exposed to BALF taken from PC mice, which showed increased levels of CD44, A549 cells exposed only to BALF showed a significant increase in cell proliferation. These results indicate that CD44-knockdown can promote cell death and aggravate lung cells from apoptosis. When the CD44 gene was knocked out in mice with PC, they showed significantly increased lung injury.

In summary, our study showed that CD44 plays an important role in regulating the inflammatory response triggered by trauma. CD44-knockdown promote cell apoptosis induced by lung injury via autophagy pathways and promote tissue damage caused by excessive autophagy activation. By revealing the mechanism by which organ damage caused by excessive autophagy is alleviated, our study has identified a new therapeutic target for the treatment of PC.

\section{Acknowledgments}

Funding: Our work was supported by the National Natural Scientific Foundation of China (No. 81772045 ) and Fundamental Research Funds for the Heilongjiang Provincial University (2018-KYYWF-0480, 2019-KYYWF-0480).

\section{Footnote}

Reporting Checklist: The authors have completed the ARRIVE reporting checklist. Available at http://dx.doi. org/10.21037/apm-20-378

Data Sharing Statement: Available at http://dx.doi. org/10.21037/apm-20-378

Conflicts of Interest: All authors have completed the ICMJE uniform disclosure form (available at http://dx.doi. org/10.21037/apm-20-378). The authors have no conflicts of interest to declare.

Ethical Statement: The authors are accountable for all aspects of the work in ensuring that questions related to the accuracy or integrity of any part of the work are appropriately investigated and resolved. The Ethics Committee of Harbin Medical University (No. IRB-AF/ SC-08/050, Heilongiiang, China) approved all experimental procedures. The procedures complied with all related state regulations. 
Open Access Statement: This is an Open Access article distributed in accordance with the Creative Commons Attribution-NonCommercial-NoDerivs 4.0 International License (CC BY-NC-ND 4.0), which permits the noncommercial replication and distribution of the article with the strict proviso that no changes or edits are made and the original work is properly cited (including links to both the formal publication through the relevant DOI and the license). See: https://creativecommons.org/licenses/by-nc-nd/4.0/.

\section{References}

1. Roberts LW, Harris PNA, Forde BM, et al. Integrating multiple genomic technologies to investigate an outbreak of carbapenemase-producing Enterobacter hormaechei. Nat Commun 2020;11:466.

2. Treskes K, Saltzherr TP, Edwards MJR, et al. Refining the criteria for immediate total-body CT after severe trauma. Eur Radiol 2020;30:2955-63.

3. GBD 2017 Cirrhosis Collaborators. The global, regional, and national burden of cirrhosis by cause in 195 countries and territories, 1990-2017: a systematic analysis for the Global Burden of Disease Study 2017. Lancet Gastroenterol Hepatol 2020;5:245-66.

4. Veysi VT, Nikolaou VS, Paliobeis C, et al. Prevalence of chest trauma, associated injuries and mortality: a level I trauma centre experience. Int Orthop 2009;33:1425-33.

5. Oyetunji TA, Jackson HT, Obirieze AC, et al. Associated injuries in traumatic sternal fractures: a review of the National Trauma Data Bank. Am Surg 2013;79:702-5.

6. Ciesla DJ, Moore EE, Johnson JL, et al. A 12-year prospective study of postinjury multiple organ failure: has anything changed? Arch Surg 2005;140:432-8; discussion 438-40.

7. Rendeki S, Molnar TF. Pulmonary contusion. J Thorac Dis 2019;11:S141-51.

8. Medar SS, Villacres S, Kaushik S, et al. Pediatric acute respiratory distress syndrome (PARDS) in children with pulmonary contusion. J Intensive Care Med 2021;36:107-14.

9. Kozan A, Kilic N, Alacam H, et al. The effects of dexamethasone and L-NAME on acute lung injury in rats with lung contusion. Inflammation 2016;39:1747-56.

10. Gaio E, Conte C, Esposito D, et al. CD44 targeting mediated by polymeric nanoparticles and combination of chlorine TPCS2a-PDT and docetaxel-chemotherapy for efficient killing of breast differentiated and stem cancer cells in vitro. Cancers (Basel) 2020;12:278.
11. Kumar S, Lanckacker E, Dentener M, et al. Aggravation of allergic airway inflammation by cigarette smoke in mice is CD44-dependent. PLoS One 2016;11:e0151113.

12. Herrington CS, Poulsom R, Coates PJ. Recent Advances in Pathology: the 2019 Annual Review Issue of The Journal of Pathology. J Pathol 2019;247:535-8.

13. Lugović-Mihić L, Novak-Bilić G, Vučić M, et al. CD44 expression in human skin: High expression in irritant and allergic contact dermatitis and moderate expression in psoriasis lesions in comparison with healthy controls. Contact Dermatitis 2020;82:297-306.

14. Luo Z, Wang Y, Lou Y, et al. Unfavorable clinical implications of peripheral blood CD44+ and CD54+ lymphocytes in patients with lung cancer undergoing chemotherapy. Int J Biol Markers 2018;33:208-14.

15. Fitzpatrick S, Lausch R, Barrington RA. CCR6positive gammadelta $T$ cells provide protection against intracorneal HSV-1 infection. Invest Ophthalmol Vis Sci 2019;60:3952-62.

16. Jang JH, Kim DH, Lim JM, et al. Breast cancer cellderived soluble CD44 promotes tumor progression by triggering macrophage IL-1beta production. Cancer Res 2020;80:1342-56.

17. Yao SY, Shen ML, Li SJ, et al. Application of a mechanically responsive, inflammatory macrophagetargeted dual-sensitive hydrogel drug carrier for atherosclerosis. Colloids Surf B Biointerfaces 2020;186:110718.

18. Yu L, Chen Y, Tooze SA. Autophagy pathway: cellular and molecular mechanisms. Autophagy 2018;14:207-15.

19. Slavin SA, Leonard A, Grose V, et al. Autophagy inhibitor 3-methyladenine protects against endothelial cell barrier dysfunction in acute lung injury. Am J Physiol Lung Cell Mol Physiol 2018;314:L388-96.

20. Qin L, Li M, Tan HL, et al. Mechanistic target of rapamycin-mediated autophagy is involved in the alleviation of lipopolysaccharide-induced acute lung injury in rats. Int Immunopharmacol 2020;78:105790.

21. Hoth JJ, Hudson WP, Brownlee NA, et al. Toll-like receptor 2 participates in the response to lung injury in a murine model of pulmonary contusion. Shock 2007;28:447-52.

22. Fei D, Meng X, Kang K, et al. Heme oxygenase-1 modulates thrombomodulin and activated protein $\mathrm{C}$ levels to attenuate lung injury in cecal ligation and puncture-induced acute lung injury mice. Exp Lung Res 2012;38:173-82.

23. Porkhanov VA, Baryshev AG, Blazhenko AN, et al. 
Treatment of patients with multiple trauma in multi-field hospital. Khirurgiia (Mosk) 2018;(12):82-5.

24. Eworuke E, Major JM, Gilbert McClain LI. National incidence rates for Acute Respiratory Distress Syndrome (ARDS) and ARDS cause-specific factors in the United States (2006-2014). J Crit Care 2018;47:192-7.

25. Loftus TJ, Kannan KB, Mira JC, et al. Modulation of the HGF/c-Met axis impacts prolonged hematopoietic progenitor mobilization following trauma and chronic stress. Shock 2020;54:482-7.

26. Zhou Y, Horowitz JC, Naba A, et al. Extracellular matrix in lung development, homeostasis and disease. Matrix Biol 2018;73:77-104.

27. Scheraga RG, Abraham S, Grove LM, et al. TRPV4 protects the lung from bacterial pneumonia via MAPK molecular pathway switching. J Immunol 2020;204:1310-21.

28. Jung YJ, Park YY, Huh JW, et al. The effect of human adipose-derived stem cells on lipopolysaccharide-induced acute respiratory distress syndrome in mice. Ann Transl Med 2019;7:674.

29. Li Z, Wu F, Xu D, et al. Inhibition of TREM1 reduces inflammation and oxidative stress after spinal cord injury (SCI) associated with HO-1 expressions. Biomed

Cite this article as: Yang S, Meng X, Yu W, Fei D, Yang W, Meng S, Luo P, Wang J, Pan S, Zhao M. Knockdown CD44 promote the inflammatory response through the autophagy pathway in mouse models of pulmonary contusion. Ann Palliat Med 2021;10(2):1456-1466. doi: 10.21037/apm-20-378
Pharmacother 2019;109:2014-21.

30. Kawaguchi M, Dashzeveg N, Cao Y, et al. Extracellular Domains I and II of cell-surface glycoprotein CD44 mediate its trans-homophilic dimerization and tumor cluster aggregation. J Biol Chem 2020;295:2640-9.

31. Wang H, Hoffman C, Yang X, et al. Targeting resident memory $\mathrm{T}$ cell immunity culminates in pulmonary and systemic protection against Brucella infection. PLoS Pathog 2020;16:e1008176.

32. Guan F, Huang T, Wang X, et al. The TRIM protein Mitsugumin 53 enhances survival and therapeutic efficacy of stem cells in murine traumatic brain injury. Stem Cell Res Ther 2019;10:352.

33. Cheng X, Zheng Y, Bu P, et al. Apolipoprotein E as a novel therapeutic neuroprotection target after traumatic spinal cord injury. Exp Neurol 2018;299:97-108.

34. Sherman MA, Suresh MV, Dolgachev VA, et al. Molecular characterization of hypoxic alveolar epithelial cells after lung contusion indicates an important role for HIF-1alpha. Ann Surg 2018;267:382-91.

35. Suresh MV, Thomas B, Machado-Aranda D, et al. Doublestranded RNA interacts with toll-like receptor 3 in driving the acute inflammatory response following lung contusion. Crit Care Med 2016;44:e1054-66. 


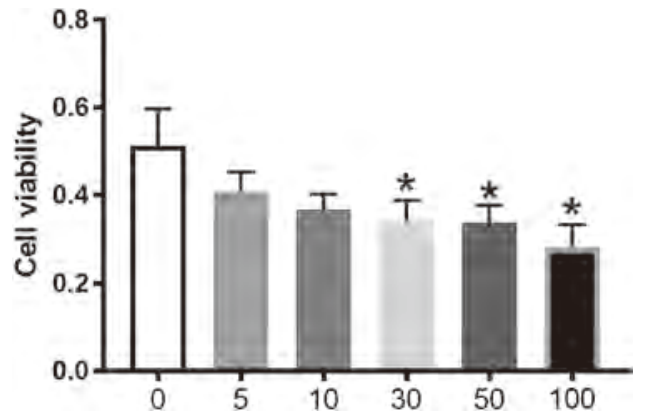

Figure S1 Viability of A549 cells exposed to BALF. *, $\mathrm{P}<0.05$ vs. the control group. BALF, bronchoalveolar lavage fluid. 